\title{
Prospective survey of investigations in outpatients referred with iron deficiency anaemia
}

\author{
A S McIntyre, R G Long
}

\begin{abstract}
Recent evidence has suggested that colonic neoplasm may be missed in patients presenting with iron deficiency anaemia unless colonic investigations are performed on all patients even when an alternative cause has been found. This study prospectively surveyed 114 consecutive patients referred from family practitioners to an outpatient clinic for the investigation of iron deficiency anaemia to determine the diagnoses contributing to the anaemia, the usefulness of certain clinical features, and the role of colonic and other investigations in obtaining the diagnosis. Upper gastrointestinal lesions contributing to anaemia were identified in 45 patients while colonic lesions were found in 18 . Twenty three patients had a non-gastrointestinal cause for anaemia and in 12 patients no cause was found. Only two patients were identified as having colonic neoplasia (a small adenomatous polyp in each case) coexisting with upper gastrointestinal lesions. Symptoms and signs had a sensitivity and specificity of upper gastrointestinal disease of $50 \%$ and $83 \%$ respectively, and $44 \%$ and $80 \%$ for colonic disease. Endoscopy had a high yield (42\%) and duodenal biopsy identified coeliac disease in three patients (two were aged $>70$ years) each of whom had normal folate values. Barium enema had a yield of $13 \%$. All colonic carcinomas occurred in patients $>65$ years. The coexistence of colonic cancer or large polyps with an upper gastrointestinal lesion identified at endoscopy was rare in outpatients referred from family practitioners. Clinical symptoms and signs were poor indicators of the investigations that will detect a cause for the anaemia. Endoscopy (with duodenal biopsy) should be performed on all patients. The yield from barium enema is so low in young patients that if an upper gastrointestinal cause is found and there are no clinical indicators it would seem unnecessary.

(Gut 1993; 34: 1102-1107)
\end{abstract}

Iron deficiency anaemia is commonly caused by chronic blood loss from the gastrointestinal tract. ${ }^{2}$ Few papers have been published on the subject since the report of Beveridge et al, which is still quoted in textbooks, ${ }^{3}$ though this study was performed before endoscopy was readily available. Lesions identified at endoscopy are now often suggested as being the cause of anaemia. More recently a series of anecdotal reports ${ }^{46}$ suggested that colonic neoplasia might be missed in patients with upper gastrointestinal symptoms or lesions. These seemed to be confirmed in an Australian study based on inpatient data. ${ }^{2}$ Sixteen per cent of patients with benign upper gastrointestinal lesions had coincidental colonic neoplasia after excluding those with partial gastrectomies and upper gastrointestinal malignancy. As a result of this study full colonic assessment seemed to be required in patients with iron deficiency anaemia regardless of the findings of upper gastrointestinal investigations. The mean age of their population, however, was high and the results cannot be extrapolated to other patient groups.

The need to investigate the colon in outpatients referred with iron deficiency anaemia has not previously been assessed. This study aimed to assess the frequency of finding unsuspected colonic neoplasia in patients with an upper gastrointestinal cause for anaemia, and also to assess the usefulness of individual investigations and clinical findings in outpatients referred with iron deficiency anaemia.

\section{Methods and patients}

The results of investigations in all patients referred by family practitioners to a medical gastroenterology outpatient clinic for the investigation of iron deficiency anaemia were prospectively collected over a 17 month period. A clinical history including the current or recent ingestion of aspirin or non-steroidal anti-inflammatory drugs (NSAIDs), iron or ulcer healing treatment was obtained and a physical examination performed. Gastrointestinal symptoms were grouped into upper gastrointestinal, which comprised dysphagia, heartburn, nausea, vomiting, upper abdominal pain (and its relief by food or antacids) or lower gastrointestinal comprising changed bowel habit, diarrhoea, constipation, rectal bleeding or lower abdominal pain (colicky, associated with changed bowel habit or relieved by passing stool or flatus). The finding of an epigastric mass or tenderness in the epigastrium was taken to suggest upper gastrointestinal disease while a mass in the right iliac fossa was taken to point to colonic disease. As gastrointestinal symptoms or signs may be non-specific two or more features related to either the upper or lower tract were considered necessary to suggest the site of possible disease.

The results of automated full blood counts (both lowest documented and on first outpatient attendance) and an assessment of iron store state (with serum iron, total iron binding capacity or ferritin, or both) were recorded. In addition serum $B_{12}$, red cell folate, coagulation studies, serum urea, electrolytes, liver function tests, and urinalysis were performed. The gastrointestinal investigations comprised sigmoidoscopy, barium enema and upper intestinal endoscopy as these are most readily available in the United 
Kingdom. In addition colonoscopy, duodenal biopsies, small intestinal radiology, ultrasound, technetium or chromium labelled studies and other non-gastroenterological investigations (for example intravenous urography) were performed when considered appropriate. Dietary iron intake was measured by a prospective seven day diary record of weighed and estimated food intake in patients who had not changed their diet before the first attendance or were not admitted for investigations to be performed.

After investigation and treatment follow up data were collected to ensure that no recurrence of anaemia occurred and that the symptoms had resolved. The mean follow up period in outpatient clinics or by contacting the family practitioner was 13.8 months (standard deviation (SD) $5 \cdot 1)$

\section{Results}

One hundred and fourteen patients were referred for the investigation of iron deficiency anaemia. Three patients were excluded from the subsequent analysis as anaemia (haemoglobin $<13 \mathrm{~g} / 100 \mathrm{ml}$ men, $<11.5 \mathrm{~g} / 100 \mathrm{ml}$ women) was not confirmed at the hospital or on reviewing the family practitioners' investigations. Sixty eight of the remaining 111 patients were women. The mean age of the group was 63 years (SD 17.3, range 20-86). The mean lowest haemoglobin was $8.6 \mathrm{~g} / 100 \mathrm{ml}$ (SD 1.9) with mean corpuscular volume $74 \cdot 1 \mathrm{fl}$ (SD 10.9).

Sixty four of the patients had received two or more weeks iron replacement before their first attendance at outpatients. None the less iron deficiency was proved in 63 of 111 patients (serum iron $<11 \mu \mathrm{mol} / 1$ with iron binding capacity $>72 \%$ or ferritin $<15 \mu \mathrm{g} / \mathrm{l}$, or both) Forty three patients had partially treated iron deficiency with a typical blood film and microcytosis (mean cell volume $<76 \mathrm{fl}$ ) or iron, iron binding capacity or ferritin, or all four at the extremes of the normal range and a response to iron treatment. Iron deficiency was not proved in five patients who were found to have duodenal ulcer, myelomatosis, giardiasis with partia villous atrophy, hepatocellular carcinoma, and menorrhagia.

The gastrointestinal investigations performed in the 111 patients included sigmoidoscopy (109), upper gastrointestinal endoscopy (108), and barium enema (92). The presence or severity of the lesions contributing to the anaemia was difficult to interpret at endoscopy in 43 of the patients as they had received a course of $\mathrm{H}_{2}$ antagonists $(n=18)$ or had reduced or stopped aspirin or NSAIDs $(n=32)$, or both, before the first hospital attendance. Duodenal biopsy specimens were taken in 50 patients. Colonoscopy was performed in 25 patients, one with ulcerative colitis as a primary investigation and in 24 to examine areas of uncertainty after the barium enema, to search for other lesions (for example angiodysplasia), or to remove colonic polyps. The colon was not assessed in nine patients in whom the diagnosis of malignant disease elsewhere had been obtained by other tests. Three patients refused endoscopy, two refused sigmoidoscopy, and nine patients refused to have a barium enema or colonoscopic examination.

UPPER GASTROINTESTINAL FINDING (TABLE I)

Endoscopy was normal or showed a hiatus hernia without oesophagitis in 66 patients. Endoscopy was abnormal in 42 patients with more than one diagnosis in many patients. Peptic ulceration, severe oesophagitis, erosive gastritis, and upper gastrointestinal malignancy were common findings. Three of the patients with normal endoscopic examinations had coeliac disease proved by duodenal biopsy (one other patient had giardia). Twenty patients had dyspeptic symptoms while receiving NSAIDs that resolved completely after reduction/cessation of NSAIDs or treatment with $\mathrm{H}_{2}$ antagonists. No other cause for anaemia was found in these patients and neither symptoms nor anaemia recurred during the follow up period. These patients may well have had NSAID induced gastrointestinal bleeding contributing to their anaemia.

\section{LOWER GASTROINTESTINAL FINDINGS (TABLE I)}

Barium enema examination showed diverticular disease in 38 patients. Colonic carcinoma was found in three patients (two caecal and one at the hepatic flexure). Barium enema also confirmed one sigmoidoscopically identified rectosigmoid carcinoma. In one case a barium enema failed to identify a carcinoma at the hepatic flexure, which was found at laparotomy six months later. Colonic adenomas were found in four cases. Concern that some areas (typically the sigmoid colon) had been inadequately visualised led to colonoscopy and the discovery of four more patients with small adenomas $(<5 \mathrm{~mm})$. Angiodysplasia of the caecum was found in one patient who also had a small adenoma.

\section{SMALL BOWEL AND NON-GASTROINTESTINAL} FINDINGS (TABLE I)

In this series a proved cause for small bowel

TABLE I The results of upper and lower gastrointestinal investigations and the non-gastrointestinal diagnosis identified in patients referred with iron deficiency anaemia

\begin{tabular}{|c|c|c|}
\hline \multicolumn{3}{|l|}{ Upper gastrointestinal findings $\left(n=45^{\star}\right)$ : } \\
\hline Gastric ulcer & 13 & \\
\hline Duodenal ulcer & 10 & \\
\hline Carcinoma & 8 & \\
\hline Oesophagitis (grade 3-4) & 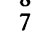 & \\
\hline Oesophagitis (grade 2) & 8 & \\
\hline Erosive gastritis & 7 & \\
\hline Coeliac disease & 3 & \\
\hline Partial gastrectomy & 2 & \\
\hline Other & 2 & \\
\hline \multicolumn{3}{|l|}{ Lower gastrointestinal findings $\left(n=18^{\star}\right)$ : } \\
\hline Colonic adenoma & 8 & \\
\hline Carcinoma & 5 & \\
\hline Ulcerative colitis & 2 & \\
\hline Angiodysplasia & 1 & \\
\hline Other & 3 & \\
\hline \multirow{2}{*}{\multicolumn{3}{|c|}{ Non-gastrointestinal diagnoses contributing to anaemia: }} \\
\hline & & \\
\hline Haematological causes & 6 & $\begin{array}{l}\text { (haemolysis } 1 \text {, } \\
\text { coagulopathy } 1, \beta \\
\text { thalassaemia trait } \\
1 \text {, blood donor } 2 \text {, } \\
\text { myeloma 1) }\end{array}$ \\
\hline $\begin{array}{l}\text { Non-gastrointestinal cancer tumour } \\
\text { Other }\end{array}$ & $\begin{array}{l}5 \\
3\end{array}$ & \\
\hline $\begin{array}{l}\text { Dietary deficiency (of } 47 \text { ) } \\
\text { No cause found }\end{array}$ & 4 & (sole cause) \\
\hline No cause found & 12 & \\
\hline
\end{tabular}

^Some patients had more than one lesion identified. 
TABLE II The relation between gastrointestinal symptoms (and signs) and the findings on investigation for upper $(A)$ and lower gastrointestinal pathology $(B)$ and for the clinical diagnosis of colonic carcinoma $(C)$. The numbers of patients with lesions and the derived sensitivities and specificities (with $95 \%$ confidence intervals (95\% CI)) are given

\begin{tabular}{|c|c|c|}
\hline \multirow{2}{*}{$\begin{array}{l}\text { Upper gastrointestinal symptoms } \\
\text { and signs }\end{array}$} & \multicolumn{2}{|c|}{ Upper gastrointestinal lesion } \\
\hline & Present & Absent \\
\hline $\begin{array}{l}\text { Present }(\leq 2) \\
\text { Absent }\end{array}$ & $\begin{array}{l}32 \\
32\end{array}$ & $\begin{array}{r}8 \\
39\end{array}$ \\
\hline $\begin{array}{l}\text { Sensitivity } \\
\text { Specificity } \\
\text { Positive predictive value } \\
\text { Negative predictive value }\end{array}$ & $\begin{array}{l}50 \% \\
83 \% \\
80 \% \\
55 \%\end{array}$ & $\begin{array}{l}95 \% \mathrm{CI} \\
38-62 \% \\
69-92 \% \\
64-91 \% \\
43-67 \%\end{array}$ \\
\hline$I$ mat or & \multicolumn{2}{|c|}{ Lower gastrointestinal lesion } \\
\hline $\begin{array}{l}\text { Lower gastrointestinal symptoms } \\
\text { and signs }\end{array}$ & Present & Absent \\
\hline $\begin{array}{l}\text { Present }(\leq 2) \\
\text { Absent }\end{array}$ & $\begin{array}{r}8 \\
10\end{array}$ & $\begin{array}{l}19 \\
74\end{array}$ \\
\hline $\begin{array}{l}\text { Sensitivity } \\
\text { Specificity } \\
\text { Positive predictive value } \\
\text { Negative predictive value }\end{array}$ & $\begin{array}{l}44 \% \\
80 \% \\
30 \% \\
88 \%\end{array}$ & $\begin{array}{l}95 \% \text { CI } \\
22-69 \% \\
72-88 \% \\
14-50 \% \\
81-95 \%\end{array}$ \\
\hline \multirow{2}{*}{$\begin{array}{l}\text { C } \\
\text { Clinical diagnosis at } \\
\text { first visit } \\
\end{array}$} & \multicolumn{2}{|l|}{ Final diagnosis } \\
\hline & Colorectal cancer & Other \\
\hline $\begin{array}{l}\text { Colorectal/carcinoma } \\
\text { Other }\end{array}$ & $\begin{array}{l}4 \\
1\end{array}$ & $\begin{array}{r}9 \\
79\end{array}$ \\
\hline $\begin{array}{l}\text { Sensitivity } \\
\text { Specificity } \\
\text { Positive predictive value } \\
\text { Negative predictive value }\end{array}$ & $\begin{array}{l}80 \% \\
90 \% \\
31 \% \\
99 \%\end{array}$ & $\begin{array}{l}95 \% \mathrm{CI} \\
28-100 \% \\
81-96 \% \\
9-62 \% \\
93-100 \%\end{array}$ \\
\hline
\end{tabular}

blood loss was an infrequent finding. One patient had ileal (by barium follow through) and rectal Crohn's disease, one had an ileal vascular malformation (showed by angiography and at laparotomy) and one had Ehlers-Danlos syndrome with a slight increase in chromium labelled red cell loss (though he also took aspirin).

The non-gastrointestinal causes contributing to the anaemia included menstrual loss (8), haematological causes (6), and malignant disease (5). Malignant disease was found in a total of 18 patients (16\%). Fifty five patients had dietary intake of iron assessed and data from 47 were evaluable. In only four patients was deficient iron intake the sole cause for the anaemia found although 27 (57\%) had dietary intakes less than the reference nutrient intake.

In 12 cases no diagnosis was made. Their anaemia did not recur over the follow up period.

MULTIPLE LESIONS CONTRIBUTING TO ANAEMIA Two or multiple causes contributing to the anaemia (excluding dietary deficiency) were found in 17 patients. In all these cases a gastrointestinal cause coexisted either with a second gastrointestinal cause (10) or a non-gastrointestinal factor (seven, including three with non-gastrointestinal malignant disease). Eleven of $18(61 \%)$ patients with oesophagitis had dual pathology. Four of $10(40 \%)$ patients with duodenal ulcers and five of $13(38 \%)$ patients with gastric ulcers also had dual pathology. Only in two of the 93 patients who had both endoscopy and colonic assessment was colonic neoplasia (in each case a small $<5 \mathrm{~mm}$ adenoma) found to coexist with an upper gastrointestinal lesion (a gastric ulcer and grade 2 oesophagitis).

\section{CLINICAL FEATURES (TABLE II)}

To find out if gastrointestinal symptoms (and signs when present) can accurately predict the need for specific investigations, symptoms were related to the endoscopic and colonic findings. Thirty four of 53 patients who had no specific symptoms or signs of gastrointestinal disease had a lesion found on investigation. Ten patients had symptoms attributable to both the upper and lower gastrointestinal tract and five had lesions in one site (four upper, one lower gastrointestinal) on investigation. Only two had both upper and lower gastrointestinal disease that might have contributed to the anaemia. Thirty two of 40 patients with upper gastrointestinal symptoms had lesions, while eight of 27 with colonic symptoms had lesions. The sensitivity for upper gastrointestinal symptoms pointing to an upper lesion was $50 \%$ with a specificity of $83 \%$. Similarly the sensitivity of colonic symptoms for colonic lesions was $44 \%$ with a specificity of $80 \%$. If colonic polyps are assumed to be asymptomatic the sensitivity of colonic symptoms predicting non-polypoid lesions rose to $69 \%$ (95\% confidence interval 44-94\%). The clinical diagnosis of colorectal carcinoma was made after the first assessment by the clinician in 13 cases; four of five cases with carcinoma and nine of 88 without carcinoma giving a sensitivity of $80 \%$ and specificity of $90 \%$.

The Figure shows the relation between the age of the patient and the identified cause of anaemia. Over half the patients in whom no cause for anaemia was found or in whom a nongastrointestinal cause (excluding malignancy) was found were less than 50 years old. The age range of the patients with benign gastric or duodenal ulceration was wide (46 to 86 years). All the upper gastrointestinal malignancies were in patients over 65 years old (range 69-87 years). Similarly all colonic carcinomas were found in patients over 65 years (range 68-80 years). Colonic adenomas were found in eight patients over 60 years (range 62-83 years). The ages of the three patients with coeliac disease were 41,73 , and 80 years.

\section{YIELD OF INVESTIGATIONS}

Table III shows the results of the investigations performed with the frequency with which they added information in making a diagnosis in the cases of iron deficiency anaemia. Serum urea, electrolyte, and liver function tests infrequently pointed to the cause of anaemia though they did identify coexisting disease. Red cell folate was normal in all cases, even in the three patients with coeliac disease. $B_{12}$ was abnormal in 12 patients (13\%). Of these two had gastric carcinomas, one had coeliac disease, one had pernicious anaemia (defined by $\mathrm{B}_{12}$ absorption studies and antibody tests), and one had a gastric ulcer. No clear cause for the $B_{12}$ deficiency was identified in the remaining seven patients. In addition three patients who already had a diagnosis of pernicious anaemia were excluded from 


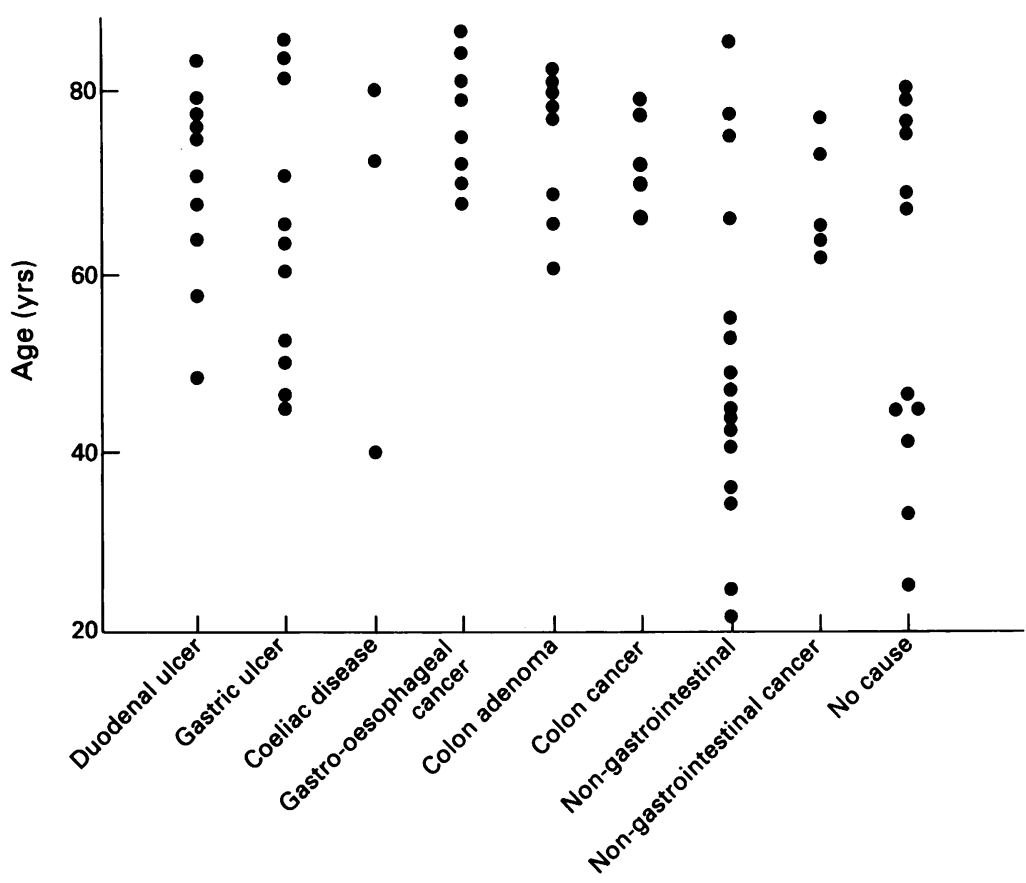

Diagram showing the age distribution of patients by diagnostic groups. The age range of patients with colonic neoplasia and other malignant disease is appreciably older than those patients with more benign disease or no identified cause of anaemia.

this analysis because they were already receiving $B_{12}$ treatment. Urinalysis and coagulation studies were seldom helpful in showing the cause of anaemia. Endoscopy contributed greatly to the diagnosis (39\%), even in cases where an alternative cause was also found. The yield of significant lesions from sigmoidoscopy and barium enema (13\%) was lower.

\section{Discussion}

This survey has audited the investigations and diagnoses in patients with iron deficiency anaemia referred to a gastroenterology outpatients by family practitioners. Previous studies have uncertain relevance to the outpatient setting as they either investigated subgroups of patients for example the elderly, ${ }^{8}$ or have reported on the findings in hospital inpatients. ${ }^{19}$ In one recent study, ${ }^{2}$ in which colonic neoplasia coexisting with upper gastrointestinal lesions

TABLE III Details of the number of each investigation performed and the contribution they added to identifying the diagnosis causing iron deficiency anaemia. Many of the 'routine investigations' have a very low yield in identifying the cause of anaemia, though some show coexisting disease for example urinalysis showing glycosuria

\begin{tabular}{|c|c|c|c|c|}
\hline Test & $\begin{array}{l}\text { Number } \\
\text { performed }\end{array}$ & $\begin{array}{l}\text { Number } \\
\text { abnormal }\end{array}$ & $\begin{array}{l}\% \\
\text { Contributing to } \\
\text { diagnosis of } \\
\text { anaemia }\end{array}$ & Notes \\
\hline Urinalysis & 105 & 7 & $1 \%$ & \\
\hline Urea and electrolyte & 107 & 7 & $0 \%$ & \\
\hline Liver function tests & 106 & 11 & $2 \%$ & \\
\hline Coagulation & 93 & 1 & $1 \%$ & APTT $53 / 27$ \\
\hline Red blood cell folate & 92 & 0 & $0 \%$ & \\
\hline & 89 & 12 & $13 \%$ & Also three on $B_{12}$ injection \\
\hline Sigmoidoscopy & 109 & 3 & $3 \%$ & \\
\hline Upper endoscopy & 108 & 45 & $42 \%$ & \\
\hline Duodenal biopsy & 50 & 4 & $6 \%$ & $3=$ coeliac, $1=$ giardiasis \\
\hline Barium enema & 92 & 50 & $13 \%$ & $\begin{array}{l}38=\text { diverticulosis, } 1=\text { cancer } \\
\text { missed at barium enema and } \\
\text { found at laparotomy }\end{array}$ \\
\hline Colonoscopy & 25 & 8 & $16 \%$ & $\begin{array}{l}16 \% \text { new diagnoses additional } \\
\text { to barium enema }\end{array}$ \\
\hline
\end{tabular}

was a frequent finding, the authors acknowledge that their results might have little relevance to outpatient practice as patients were selected by reviewing the blood counts of hospital inpatients. ${ }^{2}$ In addition the population was predominantly men and elderly. Patients referred from family practitioners are likely to be younger and might well have a different incidence of disease causing anaemia.

The frequency of upper gastrointestinal lesions (39\%) is in accord with other studies. ${ }^{210} \mathrm{~A}$ previous study has also shown a similar rate of diagnosis of coeliac disease. ${ }^{10}$ Two of the three patients who were found to have coeliac disease were over 70 years old suggesting that this diagnosis should not be dismissed because of the patients age when iron deficiency is being investigated. The absence of a low folate value should not deter the physician from obtaining a duodenal biopsy specimen. The practice of stopping NSAIDs before investigation or starting $\mathrm{H}_{2}$ antagonists for a clinical diagnosis of peptic ulceration seems to be in the patients' best interests if a delay in endoscopy is unavoidable, but may lead to failure to find a definitive cause for the anaemia. On the basis of the history, NSAIDs were implicated in 20 patients whose anaemia resolved and whose dyspepsia disappeared when the drug was stopped. No other cause for anaemia was found and anaemia did not recur during follow up. The contribution to anaemia made by mild degrees of oesophagitis or small adenomas is unclear. ${ }^{10}$ These diagnoses have been included for completeness and for consistency with previous reports. ${ }^{12}$ This study does not attempt to resolve the problem of whether such lesions contribute to anaemia. Hiatus hernia ${ }^{10}$ and diverticular disease were not considered as sufficient cause for anaemia as there is little evidence that they lead to anaemia unless complicated by oesophagitis or diverticulitis.

The comparative infrequency of colonic carcinoma that we have found compared with other studies ${ }^{2}$ is probably because of the differences in the age distribution of the populations studied. The incidence of colonic adenoma ${ }^{11-13}$ and carcinoma ${ }^{14}$ has been shown to be age related. The patients we identified with colonic cancer were in the older age range of our group with ages comparable with studies with higher incidences of cancer. ${ }^{2}$ The comparative merits of barium enema examination and colonoscopy in the investigation of iron deficiency anaemia have not been assessed in this study. Reports suggest that colonoscopy fails to reach the caecum in 17 to $45 \%$ of cases ${ }^{15-17}$ except in very experienced hands. ${ }^{18}$ As all the carcinomas were located on the right side of the colon, except the rectal carcinoma, which was found by rigid sigmoidoscopy, barium enema would seem to be an appropriate initial method of investigation. One carcinoma at the hepatic flexure was not identified on barium enema examination. Four small adenomas were missed radiologically but the importance of such lesions remains debatable ${ }^{19}$ and they are unlikely to contribute to substantial blood loss.

Previous anecdotal reports ${ }^{45}$ and one study ${ }^{2}$ have suggested that colonic investigation should 
be performed in all patients because of the frequency of dual colonic and upper intestinal disease. Our survey suggests that in outpatients the yield from barium enema examination is likely to be very low in patients in whom another cause of anaemia has been found. Only two small colonic polyps were found in patients who had an upper gastrointestinal lesion. One in a patient of 82 years who had a gastric ulcer and one in a patient with grade 2 oesophagitis, which is unlikely to have contributed substantially to the anaemia. In previous studies no clearly identifiable risk factors for colorectal cancer have been found except age (94\% of new colorectal carcinoma occurs in patients over the age of 50 years), and family history. ${ }^{14}$ Thus the routine assessment of the colon in outpatients when an alternative cause for anaemia has been found must be questioned, especially in younger patients without a family history or previous colonic polyps. None the less care must be taken in ascribing the cause of the anaemia to any single lesion. As in previous studies dual disease, which may contribute to the anaemia, was found in $20 \%$ of cases. In addition suboptimal dietary iron intake was found in many of the patients though this was seldom the sole cause for anaemia.

The sensitivities and specificities of symptoms (and signs) show that these are not good indicators of the likely site of a lesion causing anaemia. The rather low sensitivity of symptoms relating to upper gastrointestinal disease suggests that endoscopy should be performed on all patients with iron deficiency anaemia. Interestingly the clinical diagnosis of colonic carcinoma made at the first attendance was correct in four of five cases and incorrect in nine of 88 cases suggesting that clinical features are of help in this diagnosis.

This survey also allowed us to analyse the usefulness of investigations in iron deficiency anaemia in outpatients. Endoscopy has the highest yield for any of the tests performed. When performed in a patient with upper gastrointestinal symptoms it has a high probability of diagnosis. 'Routine' blood tests contribute little in finding the cause of anaemia. Serum urea and electrolytes, liver function tests, coagulation, and red cell folate were seldom abnormal and when they were it was probably because of an unrelated coexistent disease. Urinalysis by dipstick testing was abnormal in seven cases, a figure very similar to the $5 \%$ documented in unselected outpatients. ${ }^{20}$ This was mainly due to coexistent disease rather than an indicator of the cause of the anaemia.

In contrast rigid sigmoidoscopy had a small but helpful diagnostic rate, which significantly affected outcome, as two patients were found with ulcerative colitis and one patient had a rectosigmoid carcinoma. Faecal occult blood testing was not used as an entry criteria nor was its usefulness assessed during this study as the investigations to locate a possible source of bleeding would have been pursued even if faecal occult blood tests had been negative.

The coexistence of iron and $B_{12}$ deficiency has previously been recognised. ${ }^{121}$ Measurement of $B_{12}$ values showed a high rate of abnormality. As four patients had pernicious anaemia (three already on treatment), one had coeliac disease and two gastric carcinoma the checking of $B_{12}$ values in patients with iron deficiency anaemia seems to be a useful test.

Many of the differences between the findings in this study and previous ones are likely to be age related. Disease is more likely to be identified as age increases. Malignant disease, especially colonic neoplasia is rare in young subjects so investigations should be tailored to the patient's age and the clinical setting. Mandatory colonic assessment in all patients as recently suggested ${ }^{2}$ seems inappropriate.

In conclusion dual or multiple causes contributing to anaemia were present in $20 \%$ of cases of iron deficiency anaemia referred from family practitioners. Upper gastrointestinal lesions that were able to be treated were often found at endoscopy and this should be the primary investigation in such patients. The opportunity should be used to obtain duodenal biopsy specimens. Colonic assessment to detect carcinomas or polyps is warranted in older patients. The yield in younger patients is likely to be very low and the development of alternative strategies to select those in whom a barium enema or total colonoscopy should be performed would be helpful. Simple scoring of symptoms and signs is unhelpful in indicating the diagnosis, though a clinical suspicion of colonic carcinoma has reasonable sensitivity and specificity. Simple indicators of colorectal carcinoma would help to rationalise and improve the efficiency of the investigation of iron deficiency anaemia. The lack of response to iron treatment or the recurrence of anaemia when iron treatment is stopped may be an indicator of the need for further colonic assessment and requires formal evaluation. A multicentred audit under the auspices of the British Society of Gastroenterology has now started that should show more clearly the role of barium enema in the investigation of anaemia. It should also throw light on how lack of response to iron treatment points to the need for further colonic assessment.

We are grateful to the referring general practitioners, to the City Hospital radiologists and histopathologists for their help with this study, and to Mrs A Micklewright for the dietary iron assessments.

Some of this data was presented to the British Society of Gastroenterology meeting in September 1991 and published in abstract form in Gut 1991; 32: A1245.

1 Beveridge BR, Bannerman RM, Evanson JM, Witts LJ. Hypochromic anaemia. A retrospective study and follow-up of 378 in-patients. Quart $\mathcal{H}$ Med 1965; 34: 145-61.

2 Cook IJ, Pavli P, Riley JW, Goulston KJ, Dent OF. Gastrointestinal investigation of iron deficiency anaemia. $B M \mathcal{F}$ intestinal investigation

3 Pippard MJ. Iron deficiency and overload. In: Weatherall DJ, Ledingham JGG, Warrell DA, eds. The Oxford Textbook of Medicine. 2nd ed. Oxford: Oxford University Press, 1987 19: 83-7.

4 Riley JW, Wilson PC, Grant AK. Double pathology as a cause of occult gastrointestinal blood loss. $B M \mathcal{F} 1981$; 282: 626-7.

O'Reilly D, Long RG. Carcinoma of the colon presenting a dyspepsia. Postgrad Med F 1987; 63: 215-6.

6 Freestone S. Gastrointestinal investigation of iron deficiency anaemia. $B M \mathcal{F}$ 1986; 292: 1738.

7 Panel on Dietary Reference Values of the Committee in Medical Aspects of Food Policy. Dietary reference values for food energy and nutrients for the United Kingdom. London: HMSO, 1991.

8 Calvey HD, Castleden CM. Gastrointestinal investigations for anaemia in the elderly: a prospective study. Age Ageing 1987; 16: 399-404.

9 Wroblewski $M$, Ostberg $H$. Ulcer disease among geriatric inpatients with positive faecal occult blood test and/or iron inpatients with positive faecal occult blood test and/or iron 
10 Tobin MV, Gilmore IT. Gastrointestinal investigation of iron deficiency anaemia. BMF 1986; 292: 1738.

11 Arminski TC, Maclean DW. Incidence and distribution of adenomatous polyps of the colon and rectum based on 1000 adenomatous polyps of the colon and rectum based on 1000

12 Clark JC, Collan Y, Eide TJ, Esteve J, Ewen S, Gibbs NM et al. Prevalence of polyps in an autopsy series from varying incidence of large bowel cancer. Int f Cancer 1985; 36:

13 Williams AR, Balasooriya BAW, Day DW. Polyps and cancer of the large bowel: a necropsy study in Liverpool. Gut 1982; 23: $835-42$

14 Office of Population Censuses and Surveys. Cancer Statistics, Registrations. London: HMSO, 1988: Series MBI, No 16.

15 Irvine EJ, O'Connor J, Frost RA, Shorvon P, Somers S, Stevenson GW, et al. Prospective comparison of double contrast barium enema plus flexible sigmoidoscopy $v$ colono- scopy in rectal bleeding: barium enema $\mathrm{v}$ colonscopy in rectal bleeding. Gut 1988; 29: 1188-93.

16 Lindsay DC, Freeman JG, Cobden I, Record CO. Should colonoscopy be the first investigation for colonic disease? colonoscopy be the first
$B M F$ 1988; 296: $167-9$.

17 Aldridge MC, Sim AJW. Colonoscopy findings in symptomatic patients without $x$-ray evidence of colonic neoplasms. Lancet 1986; ii: 833-4.

18 Waye JD, Bashkoff E. Total colonoscopy: is it always possible? Gastrointest Endosc 1991; 37: 152-4.

19 Pollock AM, Quirke P. Adenoma screening and colorectal cancer. $B M \mathcal{F}$ 1991; 303: 3-4.

20 Morgan AG. Is routine urine testing in out-patient clinics useful? $B M \mathcal{F}$ 1988; 297: 1173 .

21 Faber K, Gram HC. Relations between gastric achylia and simple and pernicious anaemia. Arch Intern Med 1924; 34: 658-68. 